\title{
3-D Simulations of Plasma Wakefield Acceleration with Non-Idealized Plasmas and Beams
}

\author{
S. Deng, T. Katsouleas, S. Lee, P. Muggli \\ University of Southern California, Los Angeles, CA 90089 \\ W.B. Mori, R. Hemker, C. Ren, C. Huang, E. Dodd, B.E. Blue, C.E. \\ Clayton, C. Joshi, K.A. Marsh, S. Wang \\ University of California at Los Angeles, Los Angeles CA 90095 \\ F.-J. Decker, M. J. Hogan, R.H. Iverson, \\ C. O’Connell, P. Raimondi, D. Walz \\ Stanford Linear Accelerator Center, Stanford, CA 94025
}

\begin{abstract}
D Particle-in-cell OSIRIS simulations of the current E-162 Plasma Wakefield Accelerator Experiment are presented in which a number of non-ideal conditions are modeled simultaneously. These include tilts on the beam in both planes, asymmetric beam emittance, beam energy spread and plasma inhomogeneities both longitudinally and transverse to the beam axis. The relative importance of the non-ideal conditions is discussed and a worstcase estimate of the effect of these on energy gain is obtained. The simulation output is then propagated through the downstream optics, drift spaces and apertures leading to the experimental diagnostics to provide insight into the differences between actual beam conditions and what is measured. The work represents a milestone in the level of detail of simulation comparisons to plasma experiments.
\end{abstract}

\section{INTRODUCTION}

Particle-in-cell simulations have become a powerful tool for modeling plasma experiments including plasma accelerators. A number of recent works have highlighted the ability of PIC simulation to provide exquisite insight into the physics of plasma wakefield accelerators in 2-D [1-3] and 3-D [4,5]. These have been used to design new experiments [1], interpret old experiments, and test new regimes and scaling laws [6 ]. Typically, such models have used input conditions that approximate the idealized experimental conditions, such as homogenous plasmas, Gaussian beams, 
etc. In this report, we extend the way PIC models are used to a new level; namely we perform detailed modeling of the imperfections of a real experiment and an assessment of their importance. In addition, we use the simulations to provide insight into the diagnostics of the experiment. To do this, we propagate the model results out of the plasma and downstream to the diagnostic to assess the differences between what is the true state of the beam at the plasma exit and what is measured at the diagnostic.

The model we use is OSIRIS, described in Ref. 2. Except where noted, the physical parameters are that of the E-162 experiment and can be found elsewhere in these proceedings [7]. The simulation parameters were typically as follows: a computational mesh of $400 \times 100 \times 100$ grids of size $.05 \mathrm{c} / \omega_{\mathrm{p}}$ (approximately 15 microns), 9 plasma and 9 beam particles per cell, a moving window with a time-step of $.02 / \omega_{\mathrm{p}}$ run for 100,000 steps $(1.4 \mathrm{~m})$ on 64 processors at NERSC. In the next section we briefly review some modeling of non-ideal conditions of the beam and plasma separately [8]. Then we turn to the case in which all the non-ideal conditions are present at once. This is in some sense a worstcase scenario for a plasma wakefield experiment. Finally, we discuss the impact of various conditions on the performance of the wakefield accelerator.

\section{SIMULATIONS ISOLATING SINGLE NON-IDEAL EFFECTS}

\section{Non-Ideal Beams}

Figure 1 depicts the wakefields in a 3-D OSIRIS simulation for the case of round and asymmetric beams. Fig. 1a corresponds to a round beam with $4 \times 10^{10}$ electrons with a spot size of 75 microns in a plasma of density $2 \times 10^{14} \mathrm{~cm}^{-3}$. In Fig. $1 \mathrm{~b}$, the $\times 2$ (or $\mathrm{x}$ ) spot size has been doubled and the $\mathrm{x} 3$ (or $\mathrm{y}$ ) spot size has been halved. As can be seen, the amplitude of the wake is smaller when the beam is asymmetric. The reason for this is that the blow out forces [9] are different in each plane, leading to a different time for the return of electrons to the axis. The phase mixing or blur in the time for the electrons to converge to the axis reduces the amplitude of the density compression there and hence the amplitude of the wakefield spike. Despite the reduction in peak wakefield (i.e., the spike), the shape of the wake is relatively unchanged up until the spike. Thus if the wake were beamloaded ahead of the spike [6], we would expect the asymmetry to have little adverse effect. Also, the effect of asymmetry becomes less and less if the beam is made much smaller than the plasma skin depth (in both dimensions). 

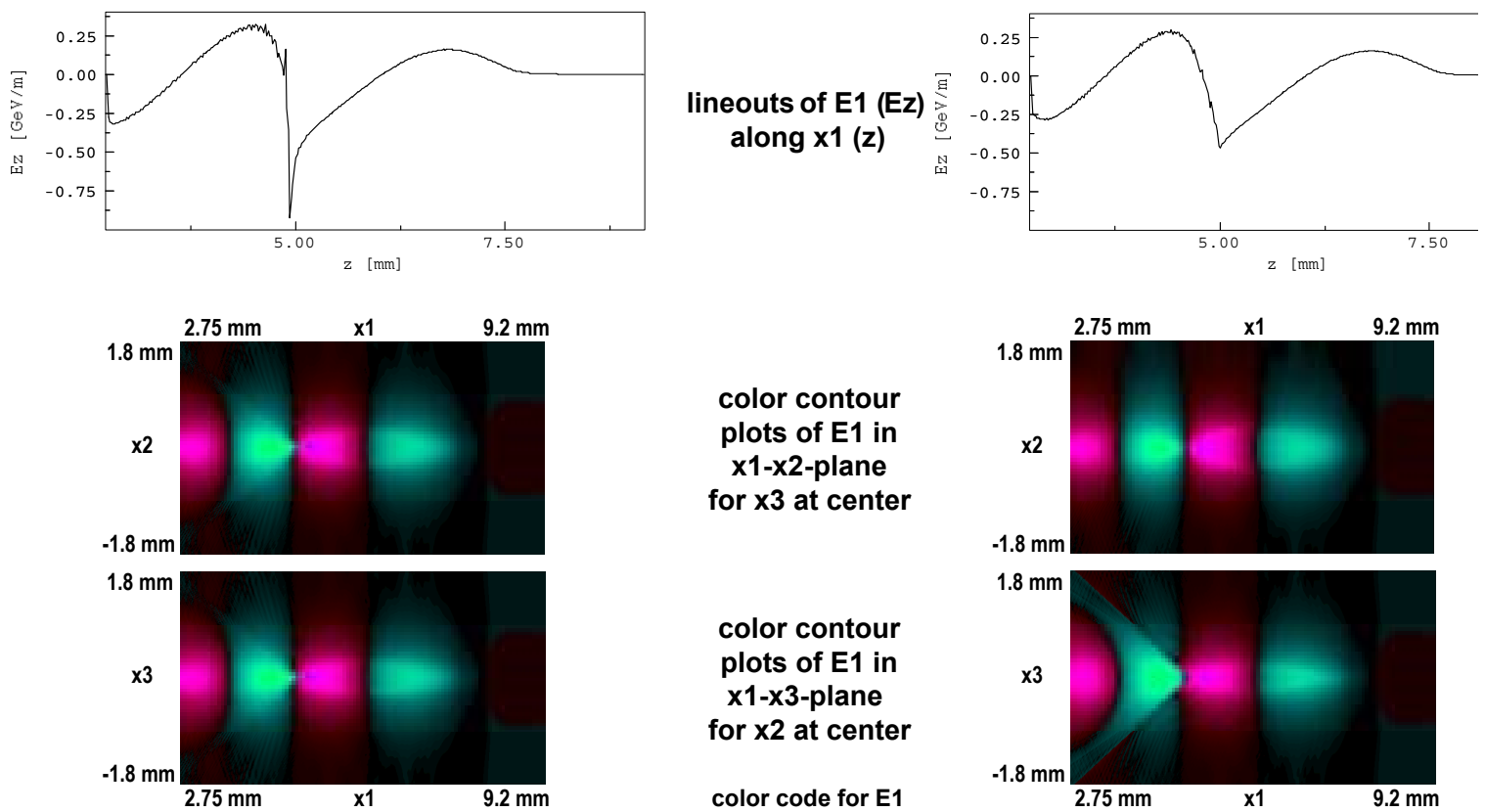

FIGURE 1. Comparison of wakes from beams with round (left) and elliptical tranverse crosssections

\section{Non-Ideal Plasma}

Figure 2 depicts a worst case image of the profile of the uv laser used to ionize the Li plasma in E-157. The hot spots lead to transverse plasma density gradients that are modeled as shown in Fig. 2b. Note the characteristic sizes of the beam and blowout channel with respect to the scale of the hot spots. The result of the asymmetric plasma is to both reduce the peak wakefield and to shift slightly the location of the peak away from the higher density plasma side. The reason for this is that the restoring force on the blown out electrons is greater on the high density side, so these electrons return to the axis first and overshoot it before meeting with the electrons from the low density side. This shift of the peak accelerating contour is apparent in Fig. 3. We note also that the area of this contour and hence the acceptance of the accelerator is less than in the homogeneous case (not shown). Finally, we mention that the focusing force becomes asymmetric in the density gradient, and this results in a deflection force on the beam. The stronger focusing on the high density side causes the beam to be deflected toward lower plasma density. Interestingly, this is opposite to the sense of the deflection of a beam near a plasma boundary (refraction) [5 ]. 


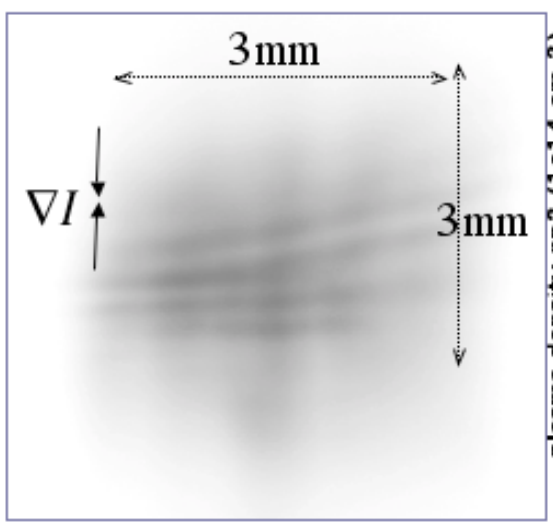

UV profile

e157(05/15/00)

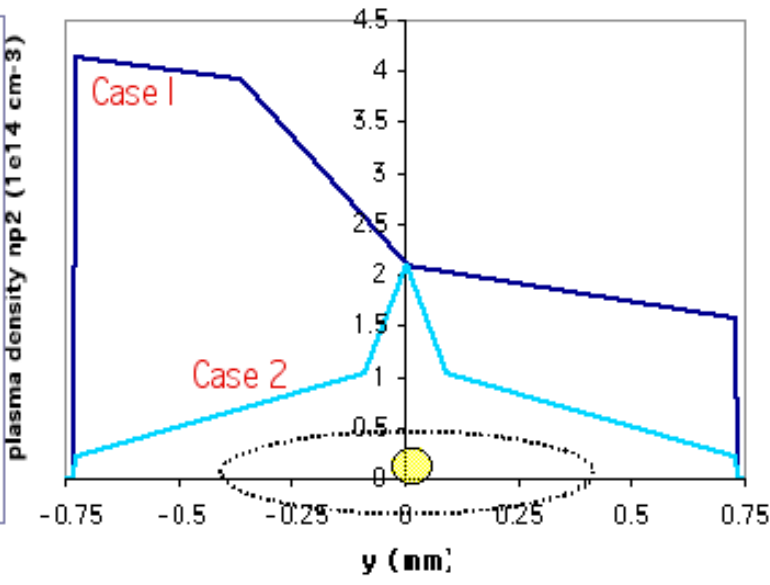

Simulation Model

FIGURE 2. Gradients in laser profile in experiments (left) and model density gradients used in simulations.

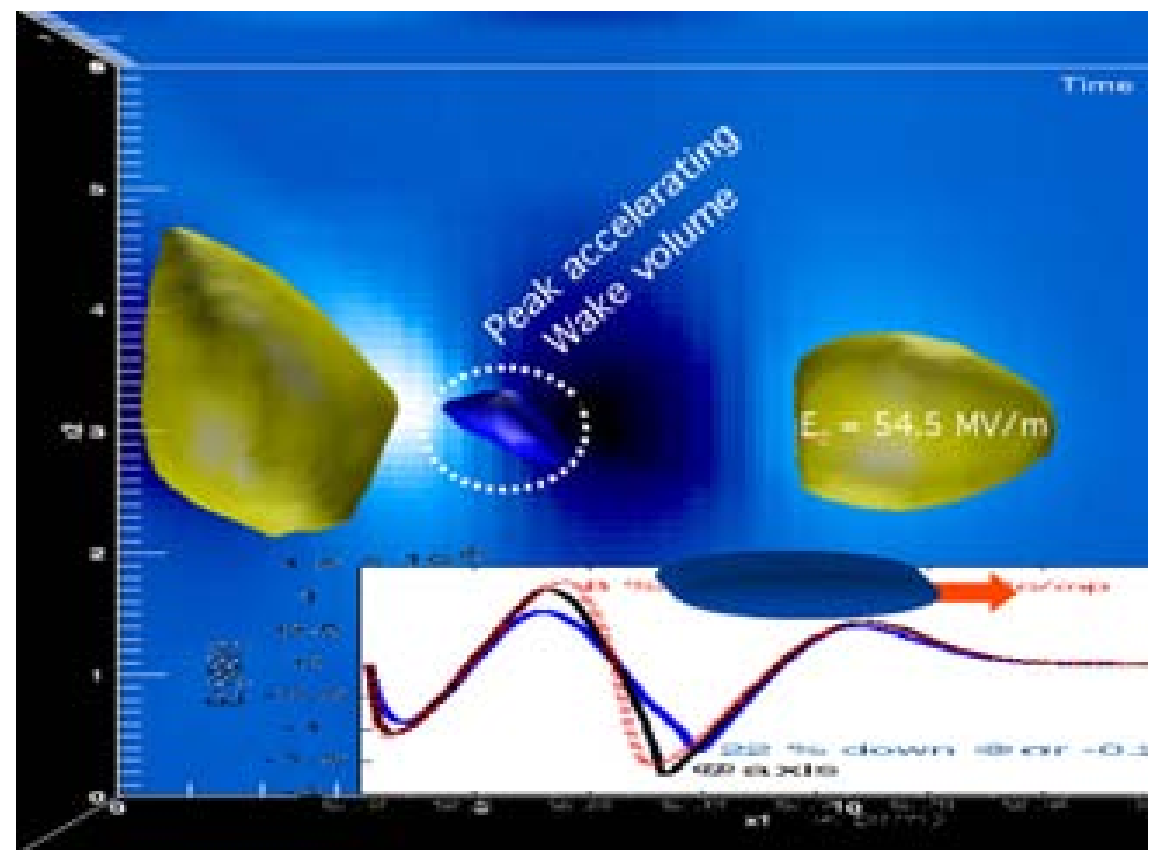

FIGURE 3. Contours of Ez in a transverse density gradient. Inset: line outs of Ez on axis at (black) and at $+-0.2 \sigma$. The peak acceleration volume is off axis toward the low density side. 


\section{SIMULATION OF SIMULTANEOUS NON-IDEAL EFFECTS: E162}

Each of the non-ideal effects discussed in the previous section yield modest reductions in the net energy gain of particles in the tail of the beam - typically $15-20 \%$ for the cases shown. When taken together, one might then be concerned that the reductions could add, resulting in negligible energy gain. To address this we present next simulation results for a case with worstcase conditions on the beam tilt, asymmetry, transverse plasma density gradient and longitudinal plasma density gradient.

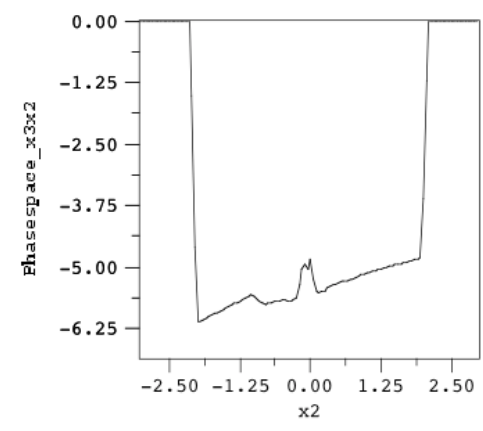

FIGURE 4. Transverse density profile in simulation.

For this case, the beam is tilted in both planes by $\sim 1 \mathrm{mrad}\left(.2 \sigma_{\mathrm{x}}\right.$ per $\left.\sigma_{\mathrm{z}}\right)$, had an uncorrelated energy spread of $.5 \%$ and unequal transverse emittances of $15 \pi$ and $60 \pi \mathrm{mm}$-mrad in the $\mathrm{y}$ and $\mathrm{x}$ directions, respectively. The initial spot size was round but evolves asymmetrically due to the different emittances. The plasma was taken to have a longitudinal density on axis that decreased from 2.3 to $1.9 \times 10^{14} \mathrm{~cm}^{-3}$ over the $1.4 \mathrm{~m}$ length (corresponding to the uncompensated pump depletion of a non-converging laser). The transverse density profile had a linear ramp increasing by $33 \%$ over the $2 \mathrm{~mm}$ system size in both planes with a "cold spot" of $10 \%$ lower density in the middle 25 microns of the simulation box (see lineout in Fig. 4).

Figure 5 shows sample output for the beam and plasma wake at early and late times in the run. The initial beam tilt is visible in Fig. 5a. At later times, a portion of the tail of the beam has been completely blown out of the simulation box. These particles were sitting just behind the electric field spike of the wake and are strongly defocused; since there is a tilt in the beam, this defocusing is asymmetric. Further back there is another group of electrons riding in the next accelerating bucket. Slices of the plasma wake show that later in the run, the location of the spike has slipped backward in the beam frame due to the longer wavelength of the wake in the low density region at the end of the run.

In Fig. 6 we show the initial and final longitudinal phase space of the beam; the energy loss of the center of the beam and the gain of the tail in relation to the energy spread can be appreciated. 

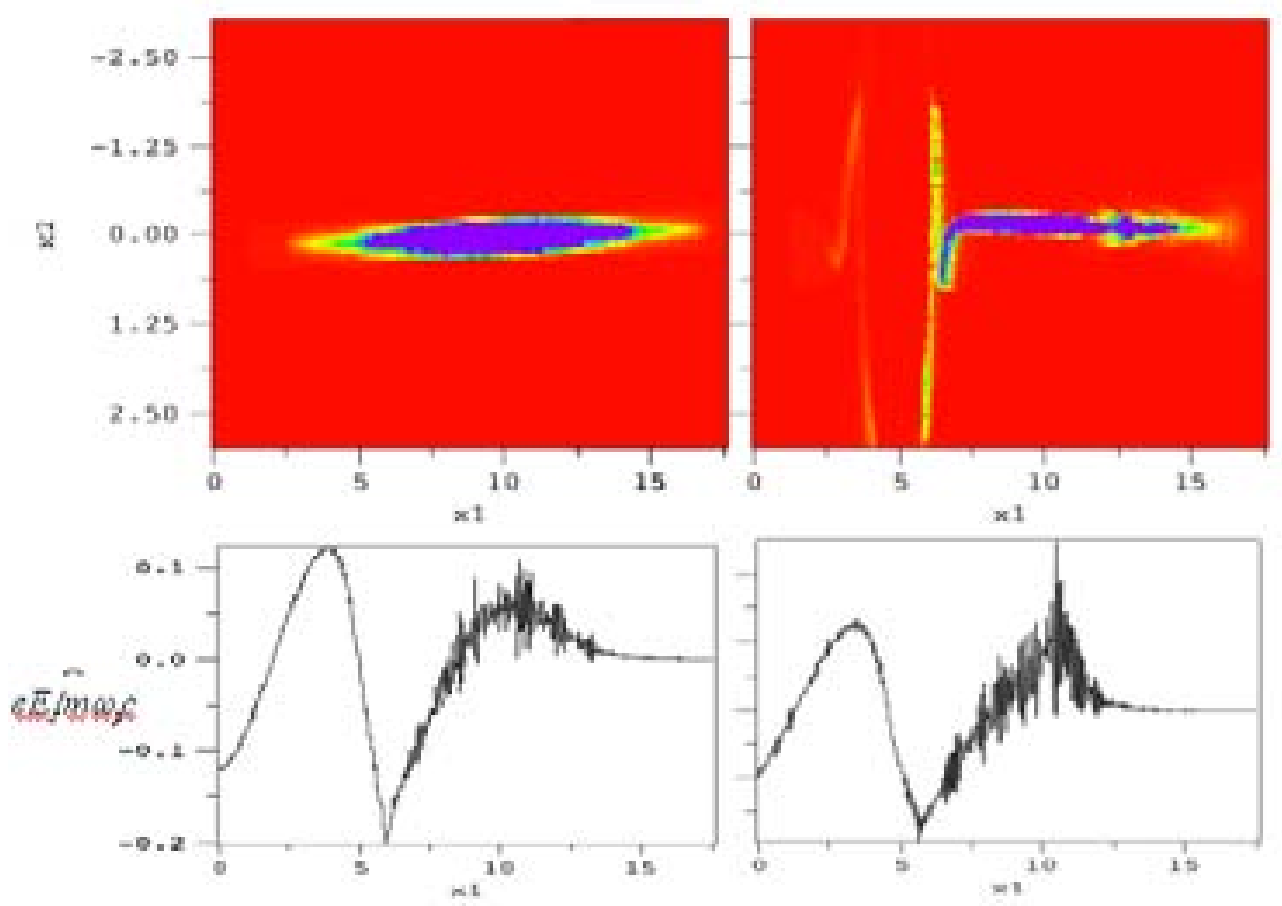

FIGURE 5. Beam profiles (top) in y-z plane at early (left) and late times in simulation in units of $\mathrm{c} / \omega \mathrm{p}$ and wakefields on axis at early and late times.

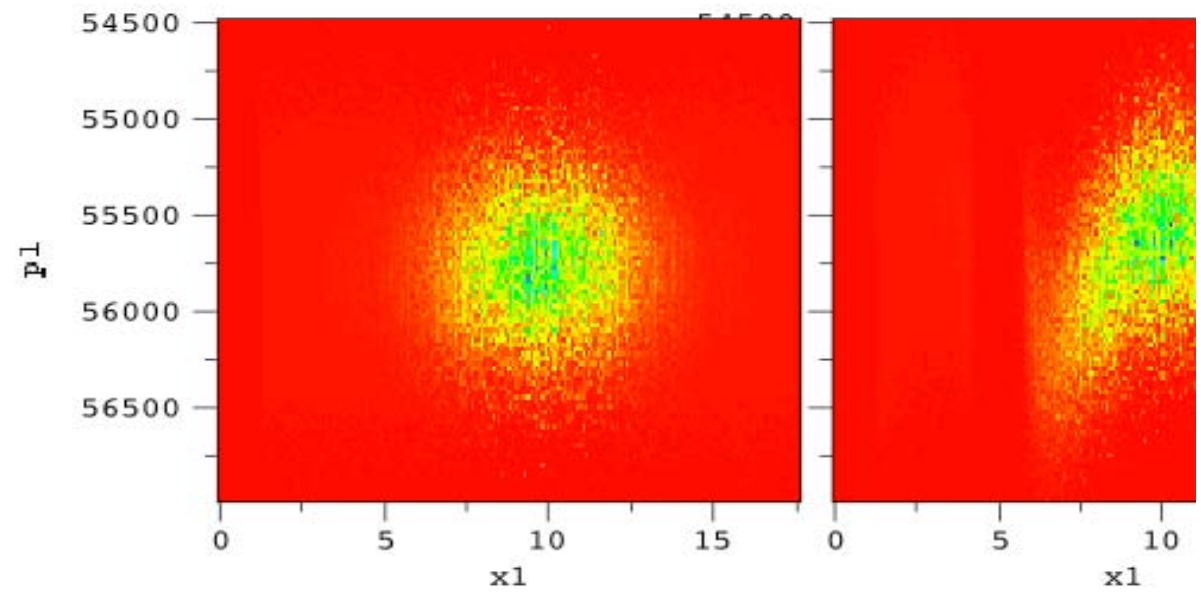

FIGURE 6. Phase space $\mathrm{P}_{\mathrm{z}} / \mathrm{mc}-\omega_{\mathrm{p}} \mathrm{z} / \mathrm{c}$ of the beam before and after the plasma in OSIRIS simulation; momentum increases downward. 


In Fig. 7 we
show the
result of
simulating
the
experimental
diagnostic for
the non-ideal
simulation
data. The
data from

Fig. 6 has been exported to the code TURTLE; a correlated spectrometer fields to the time-resolved diagnostic in E-162. Fig. 7 shows the corresponding $\mathrm{y}-\mathrm{z}$ values of the beam particles there.

Fig. 8 shows slice-average energy change of the beam in the non-ideal OSIRIS

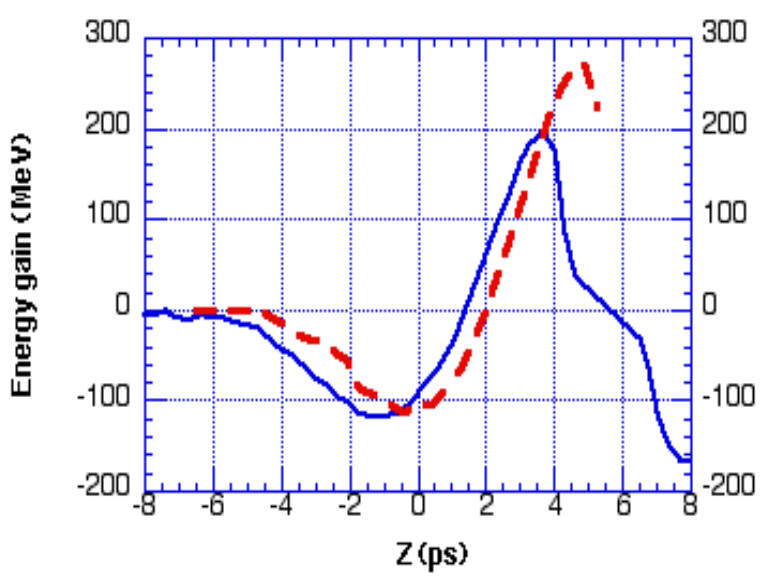

FIGURE 8. Average energy gain per ps slice for the non-ideal simulation (solid), for the ideal simulation (dashed). simulation as well as comparisons to the ideal case and to the simulated diagnostic. The energy gain of the peak slice in this case was $200 \mathrm{MeV}$ over $1.4 \mathrm{~m}$ or about $25 \%$ lower than the idealized case with no tilts and uniform plasma of density $1.5 \times 10^{14}$ $\mathrm{cm}^{-3}$. Most of the difference can be attributed to the tilt of the beam; this causes particles at the z-location of the peak accelerating field to be off axis and outside the region of peak field. Some reduction is also due to the backward slippage of the accelerating peak in the longitudinal density gradient. To simulate the experimental diagnostic, we have apertured the data in Fig. 7 in $\mathrm{x}$; this is to simulate the effect of the slit in the streak camera. The result of the beam propagation and aperture is to filter out some of the tail particles and reduce the "measured" energy gain relative the "actual" energy gain at the plasma exit. This work is still in progress and will be reported in a forthcoming paper. 


\section{ACKNOWLEDGMENTS}

Work supported by USDOE \#DE-FG03-92ER40745, DE-FC02-01ER41192, DEFG03-98DP00211, DE-FG03-92ER40727, DE-AC03-765F00515, DE-FC0201ER41179, NSF \#ECS-9632735, DMS-9722121, and LLNL \#W07405-ENG48, NSF-PHY-0078715, PHY-0078508.

\section{REFERENCES}

1. Lee, S. et al.,. Physical Review E, 61: (6) 7014-7021 Part B JUNE 2000.

2. Hemker, R. et al., Proc. Particle Accelerator Conf. (PAC), IEEE 5, 3672-4 (1999).

3. Bruwhiler, D. et al., Physical Review ST Accel. Beams 4, 101302 (2001)

2. Dodd, E.S. et al., Phys. Rev. Lett. 88, 125001 (2002) .

5. Muggli, P. et al., Nature 411, 43 May 3 (2001).

6. Lee, S. et al., Physical Review Special Topics - Accelerators and Beams, 501 (1): 1001-U9 JAN 2002.

7. Hogan, M. et al., these proceedings.

8. Ren, C. et al, in preparation.

9. Rosenzweig, J. et al., Phys. Rev. A 44, 6189 (1991). 